\title{
Design and Implementation Dynamic Website for Electronic Library
}

\author{
Halah. H. Mansoor ${ }^{1}$, Firas. A. Abdullatif ${ }^{2}$ \\ University of Information Technology and Communication, Informatics Institute for Postgraduate Studies, \\ Baghdad, Iraq ${ }^{1}$ \\ Baghdad University, College of Education for Pure Science/Ibn-Al-Haithem, Baghdad, Iraq ${ }^{2}$
}

\begin{abstract}
Dynamic an e- library is a library in which books are saved in electronic formats and attainable via computers. The e-content may be stocked locally, or arrival remotely through computer networks. An e- library is a form of information retrieval system. In the database of library, information are usually managed, collected and categorized in a way to ensure facility of access. The language was used to build e-library web application is PHP (PHP Hypertext Processor) language, and built the database by using MySQL (Structured Query Language). In this web application, the CPanel (Control Panel) of the web application used to evolve secure and dynamic web application, and to improve the Characteristics of web application.
\end{abstract}

Keywords: Library Website, Library Management, Electronic Library, Library Searching Website.

\section{INTRODUCTION}

In general Web Designing is of two categories namely Dynamic Web Designing and Static Web Designing. In Dynamic Web Designing the term Dynamic usually means capable of changing and in Static Web Designing the term Static means fixed. Static Web Designing can be applicable for small websites, but in case of large websites Dynamic Web page Designing is preferred. [1]

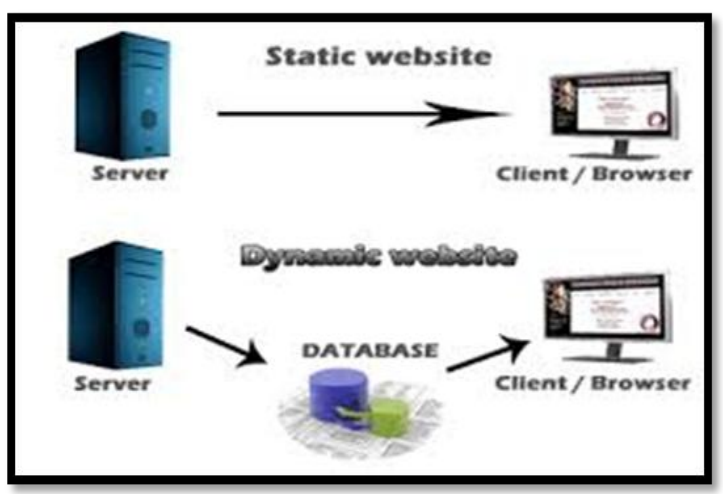

Fig. 1. Static and Dynamic website

Web pages are stored on a Web server as a number of files. A user can browse through these pages in many ways. For example, the user can type the full address of the file that contains the Web page, or the user can navigate through a Web site using the hyperlinks. As we have already discussed, the full address (called URL) of the desired Web page is embedded and associated with the hyperlink, so that when we click on the hyperlink, the address underneath is picked up and the new Web page is fetched from the server on to the client where the browser displays it There are three main types of Web pages: static, dynamic and active. [2]
Web server is special software that is replaying requests to a client (Web browser) by supplying resources (e.g. XHTML documents). For example when users insert a URL (Uniform resource locator) address into web browser they are demanding specific document from web server, the Web server maps the URL to a file in the server and replay the requested document to the client.

Using platform independent HTTP (hypertext transfer protocol) to communicate web server and the client. Use HTTP protocol for transferring requests and files over the internet (i.e. between Web server and web browser). [3] The web pages in a web site resembles an electronic library, and therefore each location of web site is like a book has a content page as its doorway to the other pages in the book, as well as all other books in the electronic library. These web pages are created using computer programming codes such as HTML, CSS, JavaScript, VBscript and many others. [4]

A digital library that includes group of digital topics which contains many resources like audio and video material, text, visual material, saved as electronic formats, in addition, means for storing, arranging, and restore the files included in the library. There are many different between digital libraries in volume and range can be kept it through organizations of the individuals, or institutions, or with academic institution. The digital data may be saved locally or remotely by computer networks. Digital libraries are large, organized collections of information objects. [5] Dynamic library management system, which provides download, search and view books to its users. Any person can use this library by enter the URL of its. Library management system is developed in PHP language and MySQL database on server side which focus on main operations in a library like adding admin, adding books, 
Vol. 4, Issue 2, February 2017

adding category, deleting books deleting category and so on. We used the HTML, java script and CSS on client side which mainly focuses on design and appearance of library system.

\section{ELECTRONIC LIBRARY DESIGN}

To design Dynamic e- library system, the database has been worked for the web application using MySQL language. The library database contains huge books data. It must be a connection between the client and server, in order to access to database information, this process can be executed by using PHP statementMysql_connect ( ) function. Database consists of one or more tables; each table consists of number of many records.After creating the database, for example "lms db", now one or more tables can be built inside the database. Figure 2 shows the library database which includesfourtables, the first table for manger's information, the second table for book's information, and the third table for categories names and the fourth table for subject names.

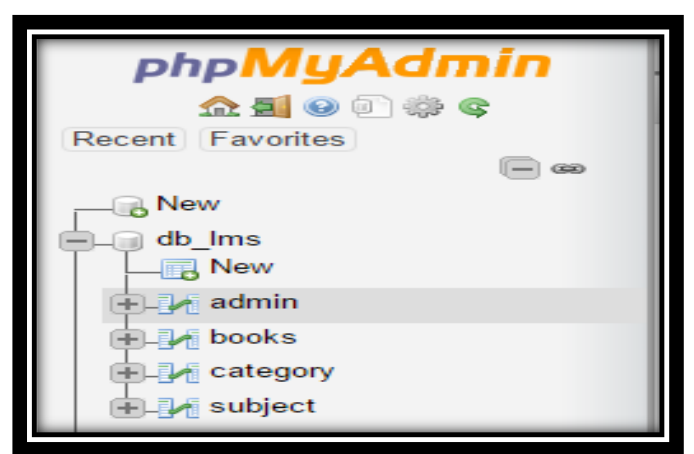

Fig.2. making database in phpMyAdmin

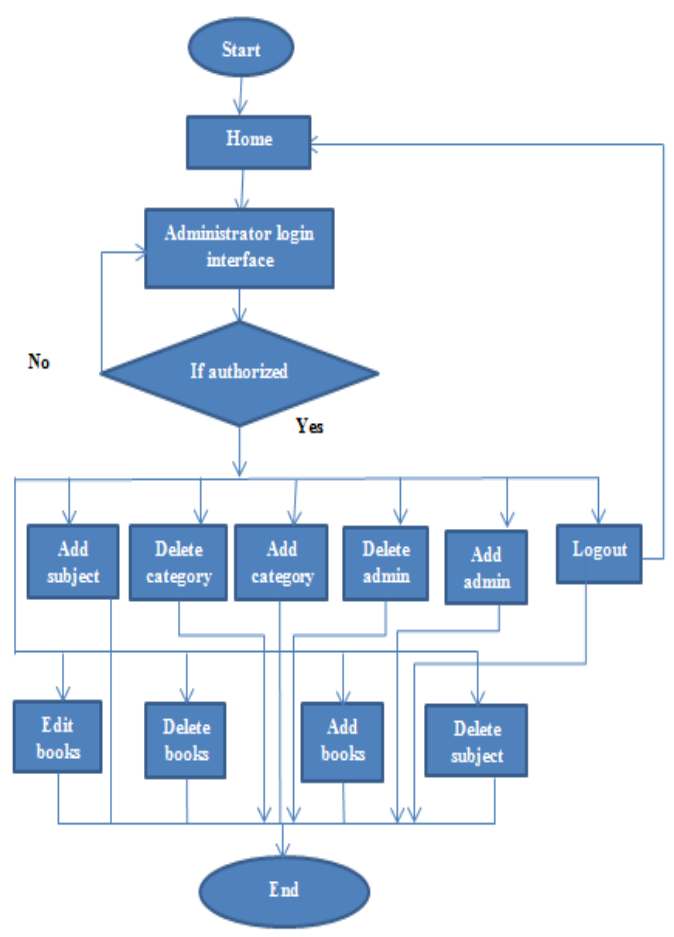

Fig.3. administrator activities diagram
The administrator can control all operations such as add, delete, edit and upload within the library web application, after recording the email and password and enter to control panel as show in figure 3 .

\section{A. Add Operation}

Manger can add categories and subjects to database by typing information in form page to be saved in category table, subject tablerespectively. The main operation in system is adding books. The manager can add a new book to the database by typing its information in the form page, to be saved in books table. The data that will received has been entered by a manager of the library within variables format that are passed to the database by the statement (Query="insert into books table the entered values"). If the information has been entered correctly, it will be stored in the database and a message will appear confirming that a new book had been keeping in the database, but if the information is not entered correctly a message is showing that the book is not saved, as shown in figure 4 .

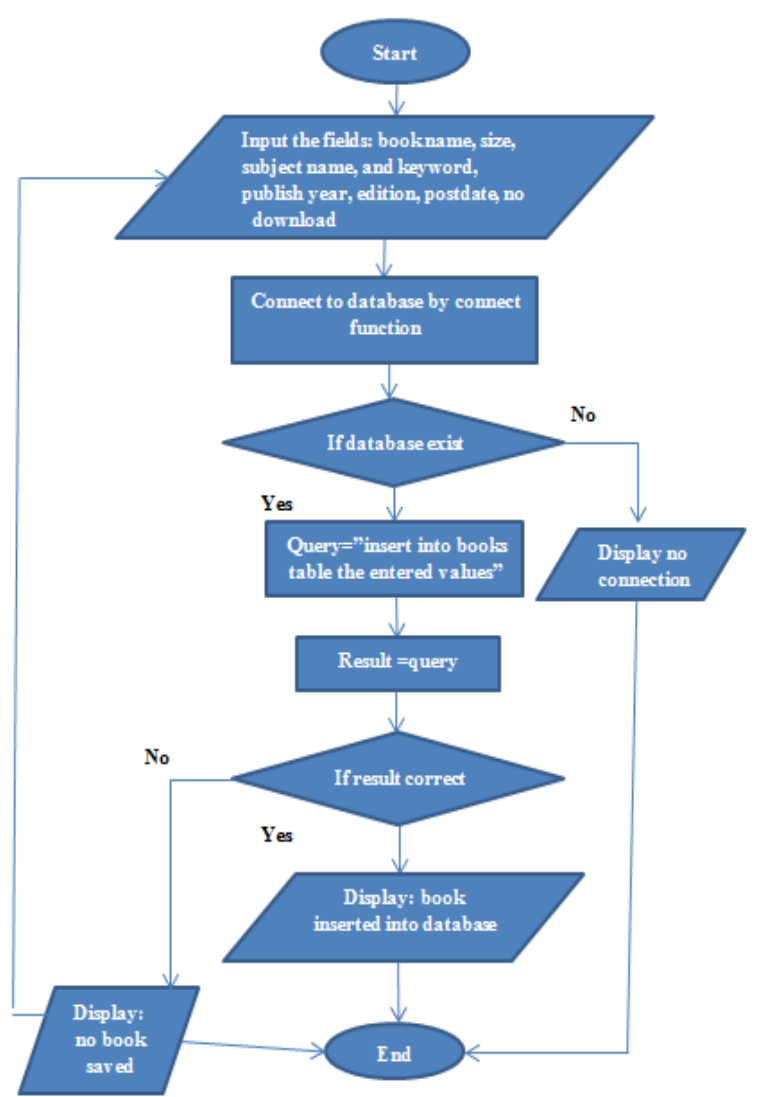

Fig.4. add book diagram

B. Delete and edit operation

Admins can delete and edit information within database, such as delete category, subject and book. When delete category all subjects and books related with these category will be deleted. And when delete subject also all books related with this subject will be deleted. If admins want to delete or edit certain book first must choose category and subject and then choose book that wanted to delete or edit it as shown in figure 5 


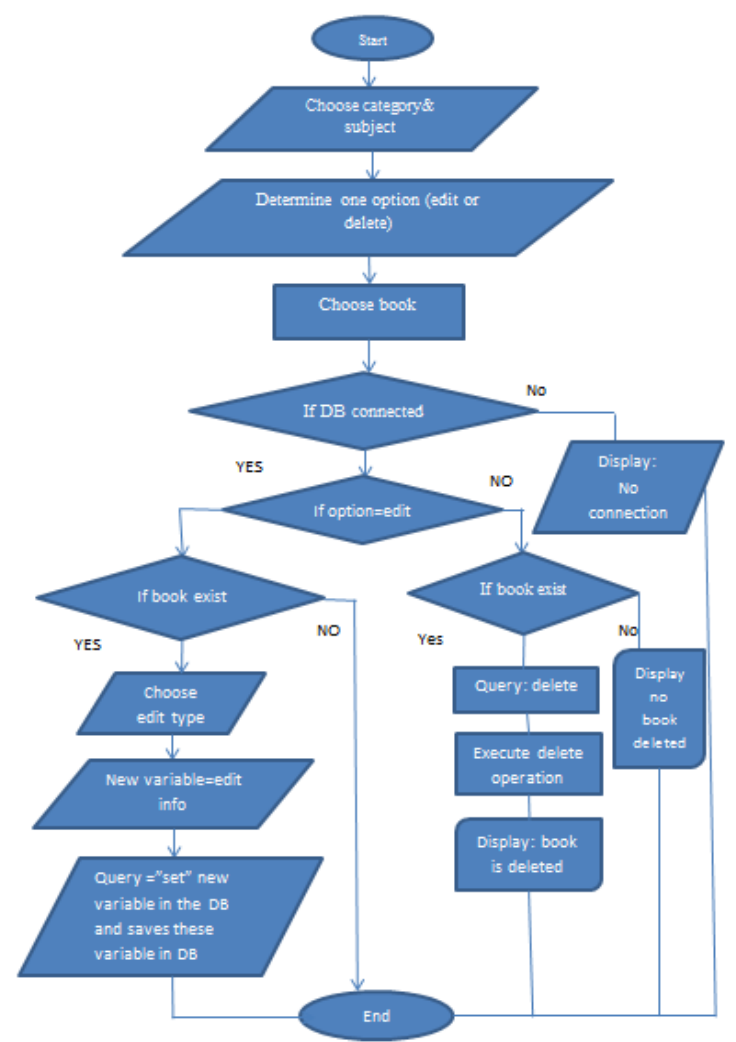

Fig. 5 edits and delete books diagram

The visitors can browse the library system, and download books, contact with library staff, and search as shown in figure 6 .

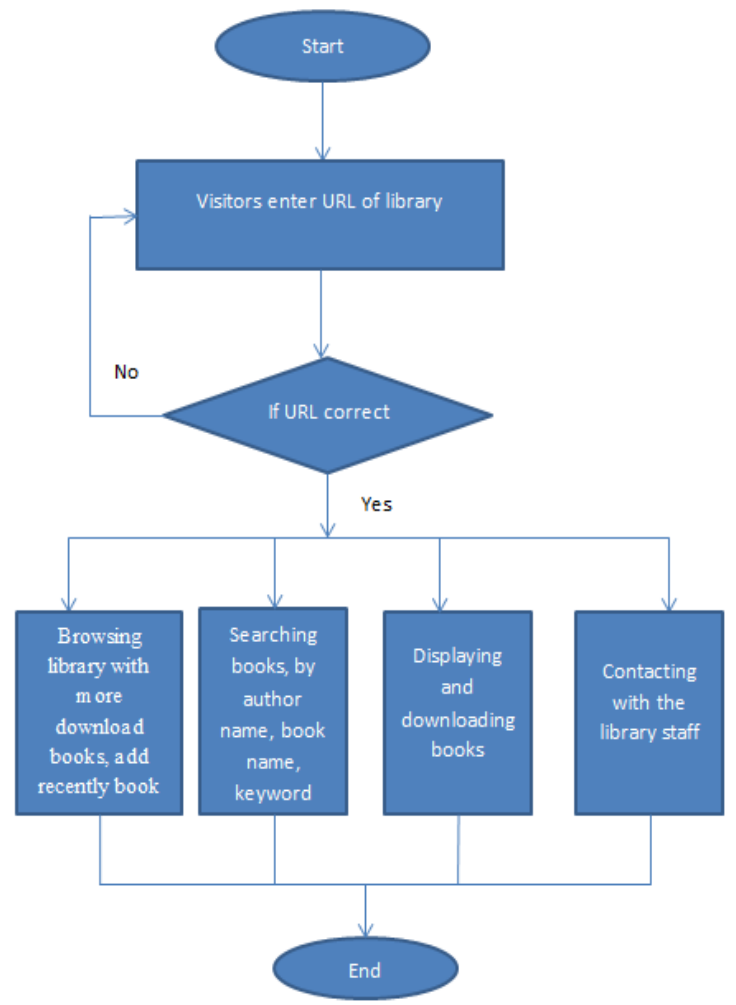

Fig. 6 users' activities diagram

\section{IMPLEMENTATION OF ELECTRONIC LIBRARY}

\section{A. Home Page Interface}

Now we will go to our implementation of Dynamic Electronic Library, we written it in PHP and MySQL to execute the operation and HTML language to be understood by web browser (Internet Explorer). This page contains a search bar at the top and horizontal menu with links such as a link to the login page, contact us page and about us page. And it contains side menu of categories names that fetch her values from the database with dropdown menu of subjects' names. And at the end of page there are more download books link, add recently book link, copyright (team work), and visitor counter. As show in figure 7

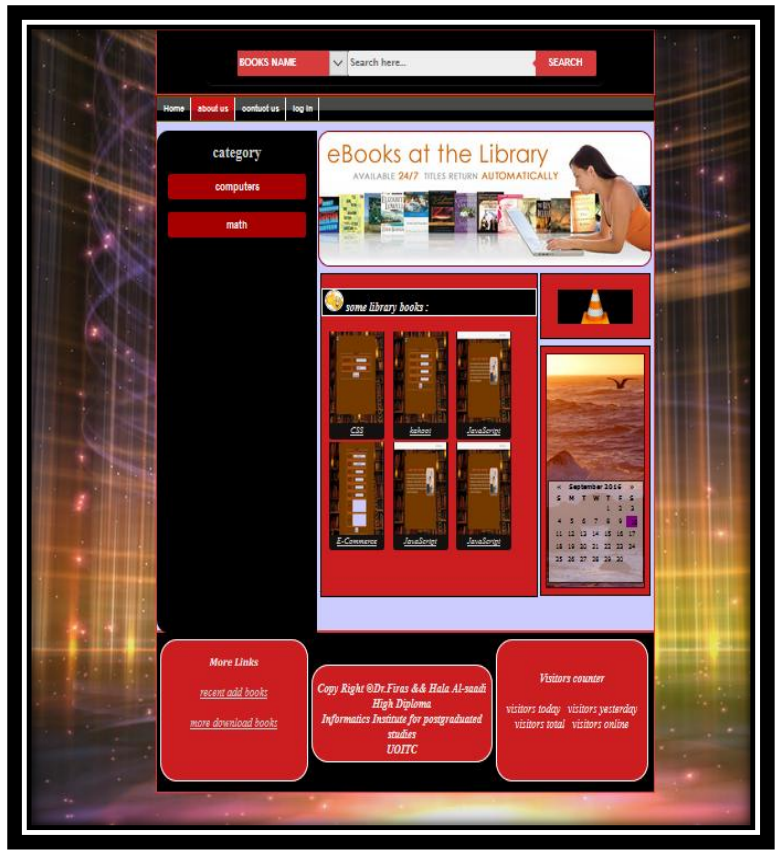

Fig. 7Home Page Interface

B. Search books:

Using the search bar at the top that users can search for a books that needs. There are three types of search the user can use them to search for a particular book, search by book's name, search by author's name, search by keywords. As shown in figure 8.

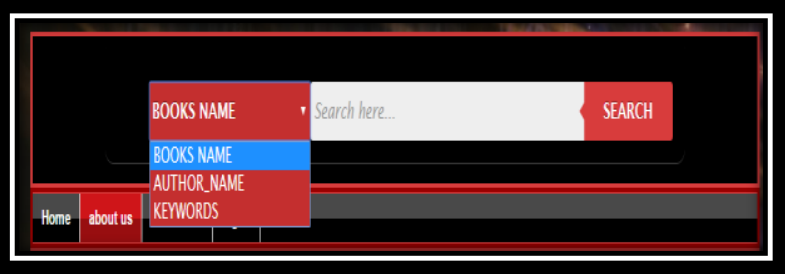

Fig.8 type of search

C. Control panel login

In this page, Admins enter their email address and password to login to Control Panel. When the Admin 


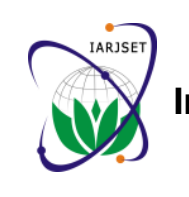

\section{International Advanced Research Journal in Science, Engineering and Technology}

ISO 3297:2007 Certified

Vol. 4, Issue 2, February 2017

enters email and password will open the session to allow him to stay in the control panel and management the whole site, where all the possibilities of additions and deletions have. The session is staying open until the admin works logout and destroy the current session. As shown in figures 9.

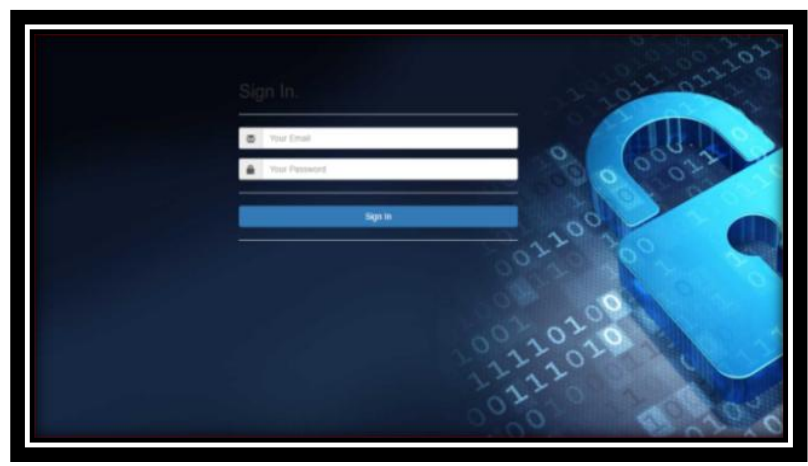

Fig 9 control panel log in

\section{Add admins}

In this page, admin can add another admins to help him to manage the system. It's containing form to enter admin's information. When submitted this form, first check if this email is already register else administrator's information are inserted in database in admins table. As shown in figure 10 .

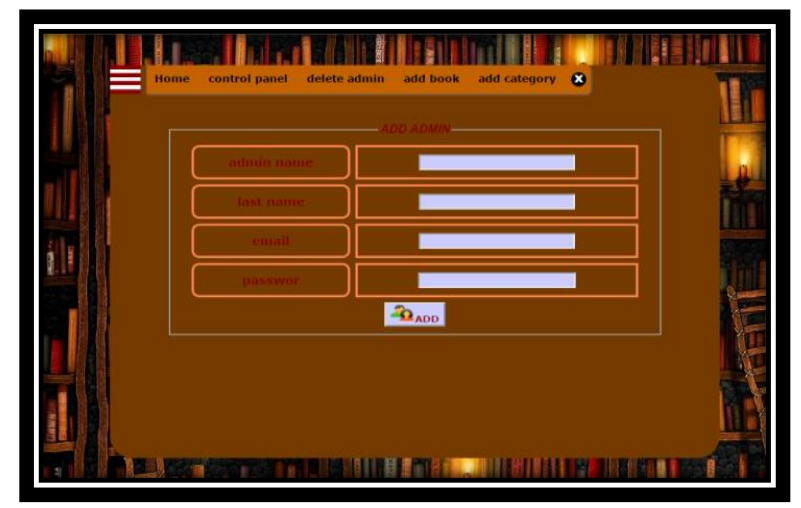

Fig.10 add admin

\section{E. Delete admins}

In this page, admins can delete another admins from the system.

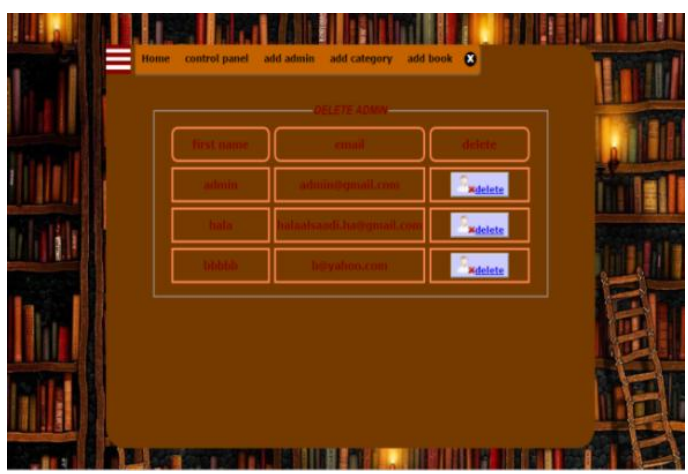

Fig.11 deletes admin
Its containing table include all admins names fetch it from database. When press deletion button will appear you message to confirm delete admin from system. As shown in figure 11 .

F. Add category

In this page, admins can add Category to the library system. When adding the category name in the text field of the form and press the add button will store the category name and id_category to database in category table.

Each category has its own subject, for example, the computer category includes artificial intelligence subject and operating system, and so on. As shown in figure 12.

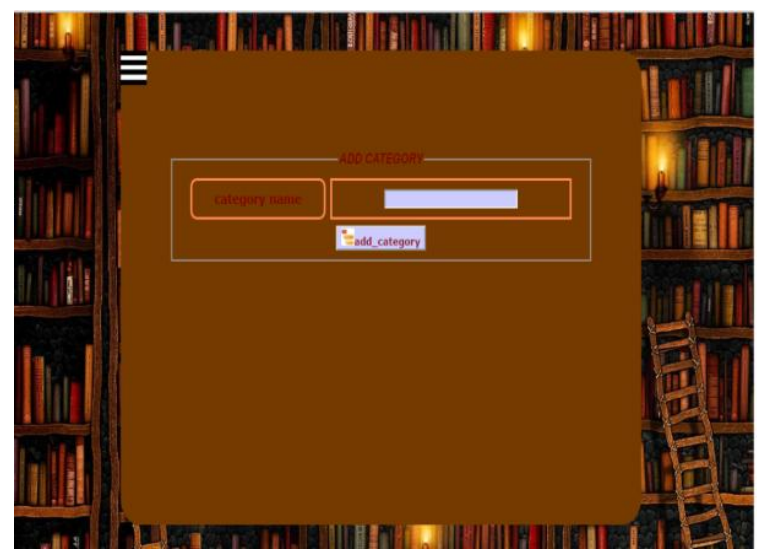

Fig, 12 add category

\section{G. Delete category}

This page contains the table of names of all of the existing categories of the library system. And by each category there are the Delete buttons. When admin presses the Delete button will show a message to confirm the deletion of this category. As shown in figure 13.

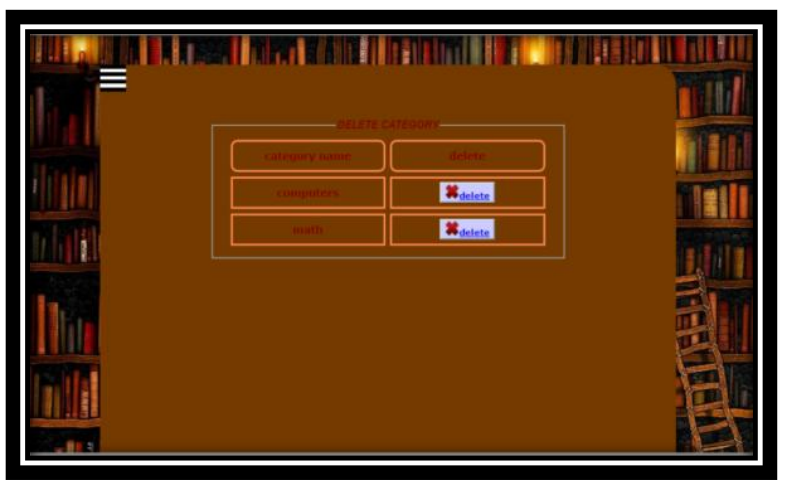

Fig, 13 delete category

H. Add subject

In this page, this admin can add certain subject to a particular category. This page contains a form inside the text field to enter the name of the subject and contains a drop-down menu that takes its values from category table. Administrator are pressed the add button to store the subject and id_category in the database in subject table. As shownin figure 14 


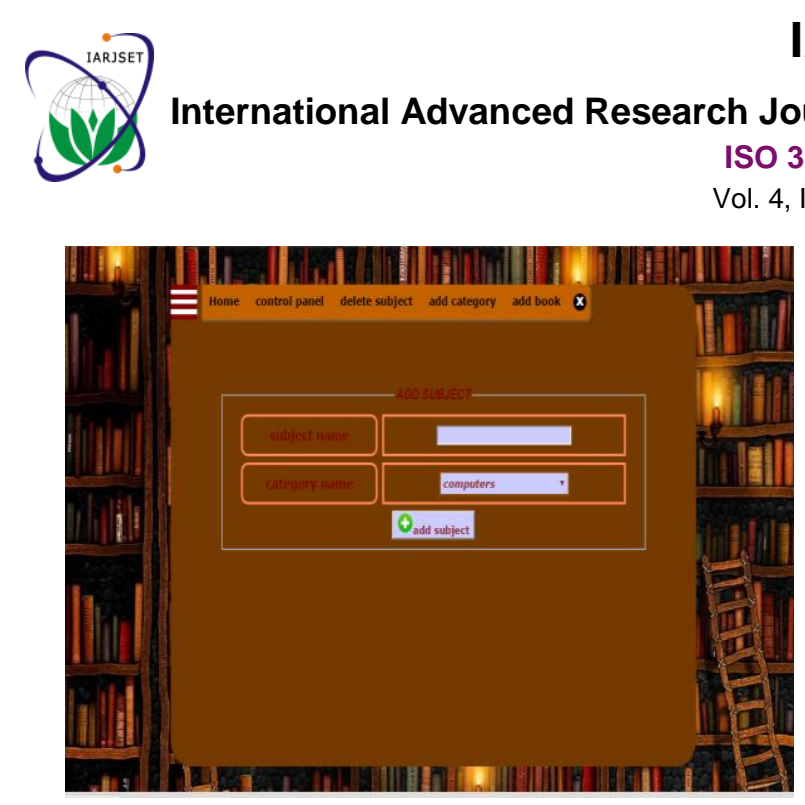

Fig.14 add subject

I. Delete subject:-

In this page, admins can delete certain subjects of a particular category. This page contains a table of the subjects that are stored in the database, there are next to each subject delete button. As shown in figure 15 .

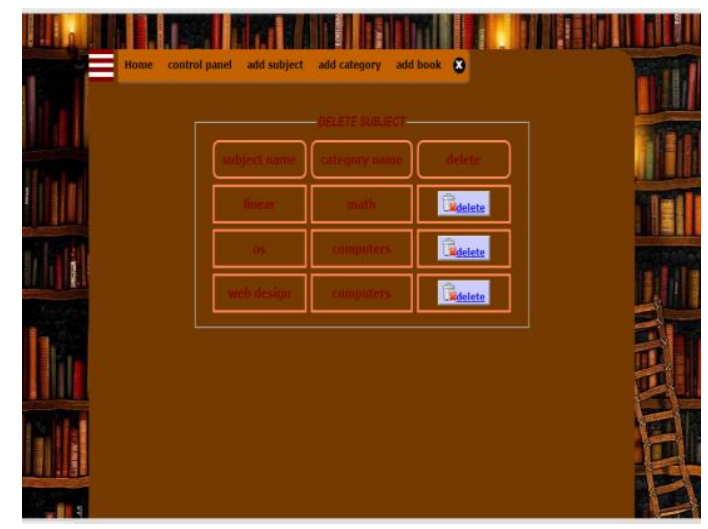

Fig.15 delete subject

J. Add books:

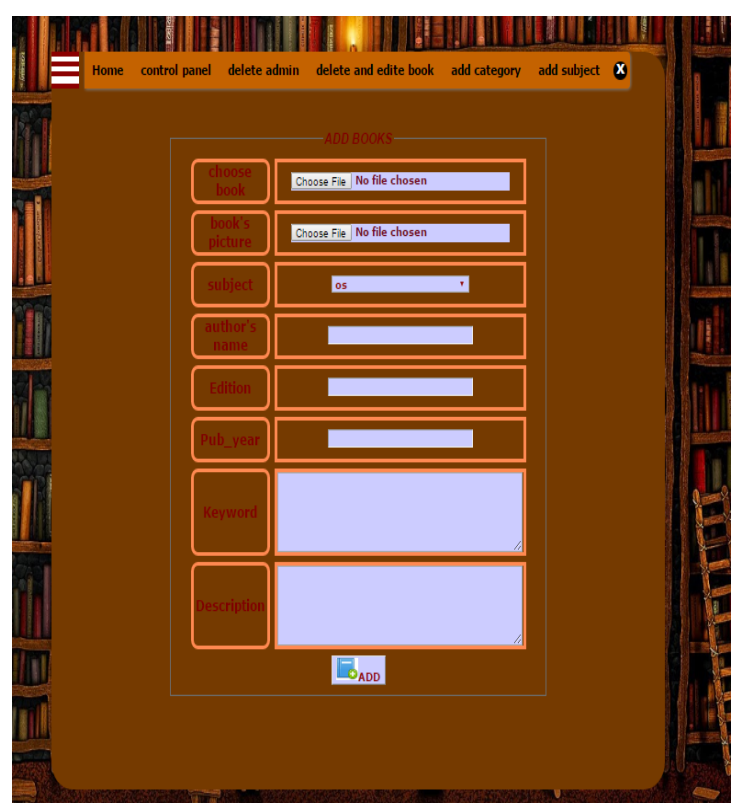

Fig 16 add book
IARJSET

:2007 Certified

In this page, admin can upload books to library system. Where this page contains a form to upload the book, Dropdown list contains the names of subject. Choose subject from the drop-down menu which will put the book under. And also contain text boxes to enter the name of the author, edition of the book and the year of publication and also contains a text area to enter keywords for the book that will help us in the search for particular book. When you press the button add, books information is stored in the database, while the book is loaded to a folder within the site. As shownin figure 16.

\section{K. Delete books}

In this page, admin can delete books from the library system. To delete a specific book must first choose the category and the subject that we want to delete the book of them as shownin figure 17.a. When selecting a particular subject within a certain category and press on the view button will appear all books of this subject in other page. When pressing the delete button, will delete the book from system. As shown in figure 17.b.

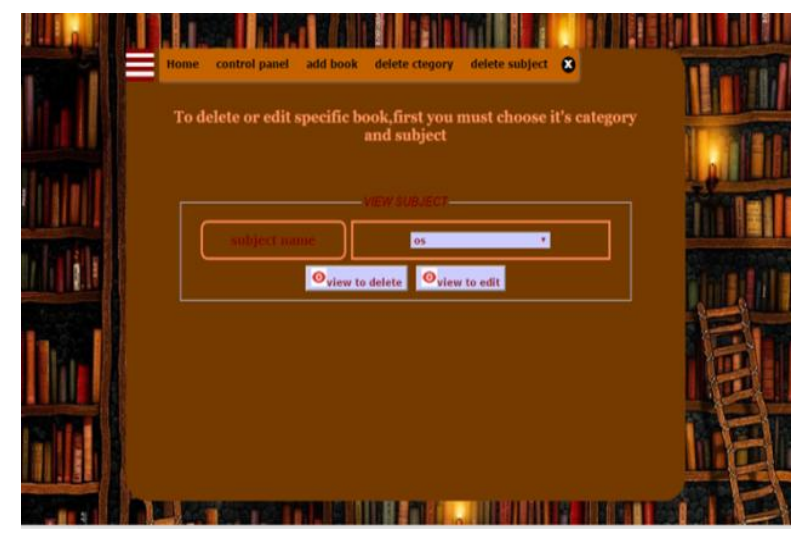

Fig. 17.a delete book (choose subject).

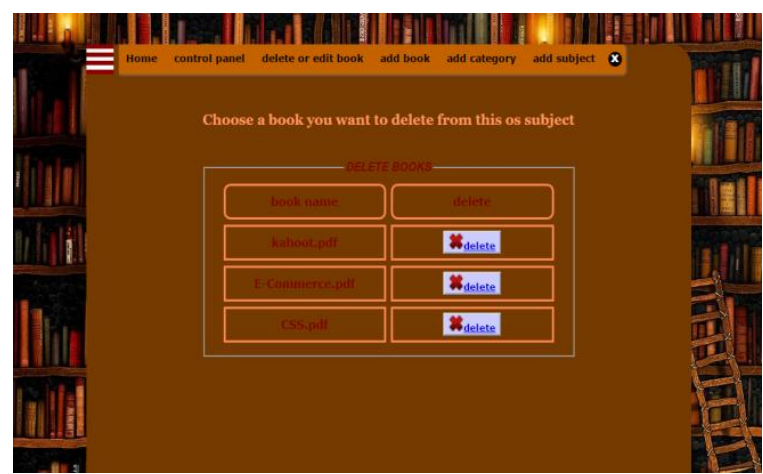

Fig. 17.b delete book

L. Edit books:-

Admin can edit a particular book in the system .first admin must choose the category and subject that wants to edit the book from him as shownin figure 18.a. When choosing a particular subject within a particular category and click on the view button will show all the books of the subject, as shown in figure 18.b. when pressing the edit button will move to another page to update book information. As shown in figure 18.c. 


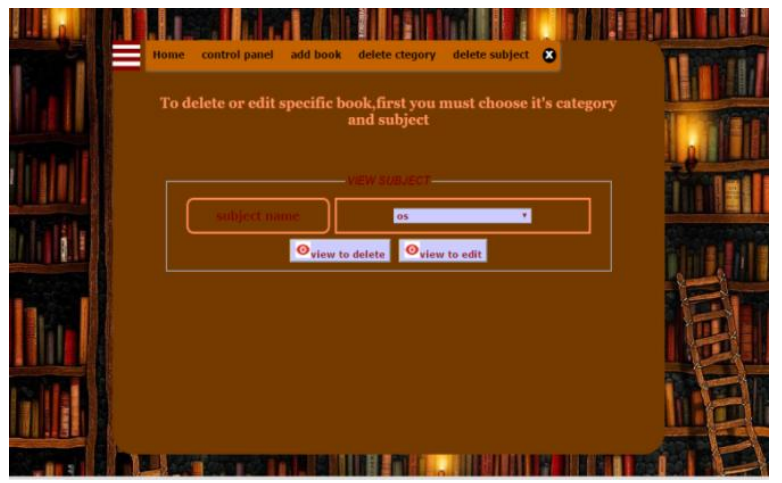

Figure 18.a edit book (choose subject)

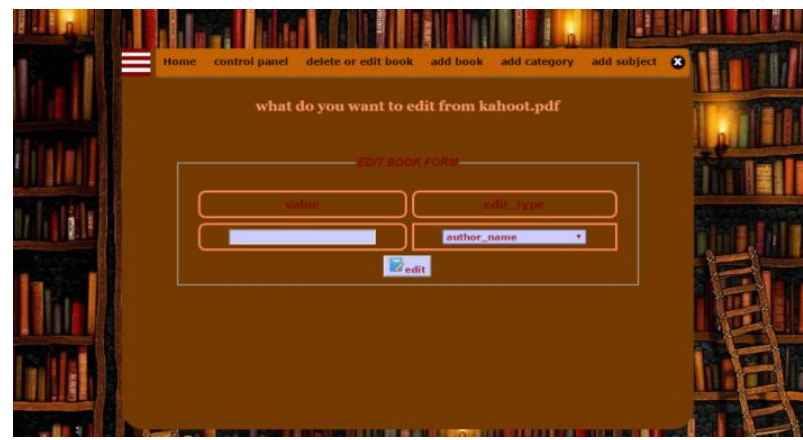

Fig18.b edit book (choose book).

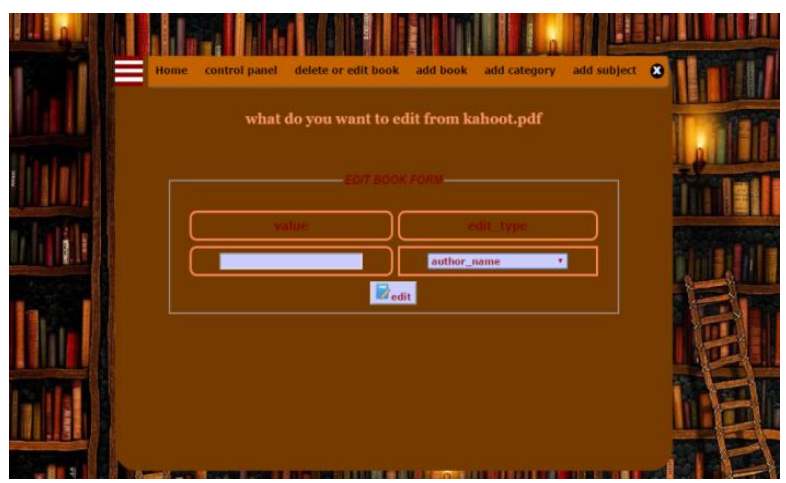

Figure 18.c edit book

\section{CONCLUSION}

One of the most important features on using server side scripting is browser independent; that will also make source code safer from being accessed by anyone. MySQL database which supply the mechanism to store, retrieve data and give the permissions to any user as it is planned by the system administrator, and provides flexibility. When designing web site; one important issue to perform is site's security, by applying user authentication, restricting web pages access, and preventing direct database access.

\section{ACKNOWLEDGMENT}

I would like to thank Dr. Firas A. Abdullatif for his supervision during the period of the research, encouragement, comments and help, thanks all those who stand beside me in my study and give me the encouragement to do this research, Family, and supportive friends.

\section{REFERENCES}

[1] http://www.phonatetech.com/dynamic-website-development, accessed 22-9 2016.

[2] Godbole, A.S., and Kahate, A. "Web technologies : TCP/IP to Internet application architectures' (Tata McGraw-Hill, 2003. 2003)

[3] Sean, W.: 'An Introduction to web sites Basics', 1998.

[4] Deitel, H.M.: 'Internet and World Wide Web: how to program' (2002. 2002).

[5] Witten, I.H., Bainbridge, D., and Nichols, D.M.: 'How to Build a Digital Library' (Elsevier Science, 2009. 2009). 